Tuchschmid: Einfluss der Temperatur etc. 235 schaften sind vielfach andere. Die Chemie erforscht die Eigensehaften der Elemente und ihrer Verbindungen durch fortwährende Darstellungen neuer Species, neuer künstlich combinirter Species, die meist in der Natur nicht vorkommen, oft auch unter dem Einfluss der allgemein waltenden Agentien der Luft, des Wassers, des Temperaturwechsels etc. gar nicht vorkommen oder bestehen könnten. Die Mineralogie beschäftigt sich nicht mit der Darstellung neuer Species, sie hat es auch nicht mit Abkömmlingen aus dem organischen Reich zu thun, sie hat die ursprünglich unorganischen Species, welche in der Natur vorkommen, zum Gegenstand des Studiums, und da haben sich für die Darstellung der Mischungen die bisher gebrauchten Formeln bewährt, und geben einfacher, unmittelbarer und bestimmter an als die modernen, was aus einer solchen Formel zu ersehen sein soll. ${ }^{1}$ ) Wenn erwähnt wird, es wiirden die Mineralogen, wenn sie ihre Formeln beibehalten, von der jüngeren Generation der Chemiker nicht mehr verstanden werden, so scheint mir dieses Bedenken nicht erheblich, denn wenn diese Generation Mineralogie treiben will, so wird sie anch die Formeln verstehen lernen, welche man in der Mineralogie für die zweckmässigeren hält.

Ueber den Einfluss der Temperatur auf das molekulare Drehungsvermögen einiger circular polarisirender Substanzen;

Von

\title{
Dr. C. Tuohsohmid.
}

(Hierzu Tafel I-VI.)

Seitdem Biot ${ }^{2}$ ) in der Mitte der Dreissigerjahre die Gesetze der Drehung der Polarisationsebene durch Zucker-

1) Dies. Journ. 103, 159.

7) Ann. Chem. Pharm. 52, 186. 
lösungen und die Lösungen einiger anderer Substanzen studirt und bekannt gemacht hatte, wurden verschiedene Instrumente in die Technik eingeführt, am, gestuitzt anf die Stärke der Drehung des polarisirten Lichtstrahles, die man mit Hülfe der Instrumente messen konnte, namentlich in Zuckerlösungen den Gehalt derselben an reinem Zucker zu ermitteln.

Bei der grossen Wichtigkeit, welche diese Polarisationssaccharimeter alsbald in der Zuckertechnik erlangten, konnte es nicht ausbleiben, dass die von Biot erkannten Gesetze von verschiedenen Forschern nach allen Seiten hin geprüft und ergänzt wurden, um auf diese Weise alle Momente festzustellen, welche störend oder modificikend auf das Resultat der Untersuchungen einwirken könnten. In Folge dessen wurde schon früh die Abhängigkeit des molekularen Drehungsvermögens von der Temperatur beobachtet. Schon im Jahre 1842 veröffentlichte Mitscherlich ${ }^{1}$ ) in einer Abhandlung, dass bei schwachem Erwärmen oder Abkühlen einer Rohrzuckerlösung ihr Drehungsvermögen nicht geändert wird, dass aber das Drehungsvermögen von Fruchtzuckerlösungen wesentlich von der Temperatur beeinflusst werde. Die gleiche Thatsache bestätigten später Ventzke') und Dubrunfaut ${ }^{8}$ ), ohne dass jedoch einer der gonannten Forseher das Gesetz, nach welchem diese Veränderungen des Drehungsvermögens mit der Temperatur vor sich gehen, näher studirt hätte.

Erst Clerget4) gebührt das Verdienst, diesen Einfluss der Temperatur auf das Drehungsvermögen invertirter Zuckerlösungen festgestellt zu haben, seine Beobachtungen geschahen mit Hülfe des Soleil'schen Saccharimeters, das bei der Anwendung der Doppelplatte und des Quarzcompensators eine bedentend genauere Einstellung erlaubte, als das frihere Mitscherlich'sche Instrument. Clerget

1) Berl. Akad. Ber, 1842, S. 150.

2) Dies. Journ. 28, 101.

3) Ann. ch. phys. [3] 18, 99.

4) Ann. ch. phys. [3] 26, 175 und Ann. Chem. Pharm. 22, 145. 
auf das molekulare Drehungsvermögen etc. 237 benutzte seine Beobachtungen zur Herstellung einer 'Tabelle, die aus der Drehung einer Zuckerlösung vor und nach der Inversion, und der Temperatur, bei welcher die Beobachtung gemaoht wurde, unmittelbar den Gehalt der zu untersuchenden Flüssigkeit an reinem Zueker angiebt. Die neuesten Untersuchungen auf diesem Gebiete stammen von $\mathrm{Pohl}^{1}$ ), welcher die Abhängigkeit des Drehungsvermögens von Fruchtzuckerlösungen von der Temperatur für das Mitscherlich'sche Instrument bestimmte.

Clerget erwähnt in seiner Abhandlung, dass eine Lösung von 16,471 Grm. reinen Zuckers in $100 \mathrm{Cc}$. Wasser die Polarisationsebene um 100 Theilstriche der Soleil'schen Scala ablenke, wenn man diese Lösung in einer Röhre von $200 \mathrm{Mm}$. beobachtet, dass diese gleiche Lösung nacb der Inversion die Polarisationsebene im entgegengesetzten Sinne um $44^{\circ}$ drebe, vorausgesetzt dass man die Beobachtung bei $0^{\circ}$ vorgenommen hat, dass die Drehung mit der Temperatur abnehme und zwar bei $\mathrm{Zu}-$ nahme um je $1^{\circ}$ um 0,5 Theilstriche. Auf diese Beobachtung gründet $\mathrm{Clerget}$ seine Tabelle.

Als später von verschiedenen Autoren gezeigt warde, dass nicht eine Lösung von 16,471 Grm., sondern von $16,35 \mathrm{Grm}$. reinen Zuckers in der erwähnten Menge Wasser gelöst und in der Röhre von $200 \mathrm{Mm}$. untersucht, die Polarisationsebene um $100^{\circ}$ ablenkt, wurde in der entsprechenden Weise die Clerget'sche Tabelle ungerechnet. Jedenfalls können die von Clerget gemachten Angaben über das Drehungsvermögen invertirter Zuckerlösungen und ihrer Aenderungen mit der Temperatur nur Näherungswerthe aein; es erklürt sich wohl hierans, dass die Inversionsmethode den Rohrzuckergehalt bis $\mathrm{zu} \pm 0,4$ p.C. schwankend angiebt, wie dies Landolt ${ }^{2}$ ) nachgewiesen hat; Fehler, die sich nicht allein aus den möglichen Beobachtungsfehlern herleiten lassen, da diese nach den gleichen Untersuchungen bedeutend geringer ausfallen.

1) Wien. Akad. Ber. 1856, S. 492.

*) Bericht über chem. Zuckeranalysen von Prof. Landolt. 1868. 


\section{Tuchschmid: Einfluss der Temperatur}

Da aber bei Zuckerproben die Inversionsmethode immer in Anwendung gebracht wird, um so zu erfahren, ob diese keine Substanzen enthalten, die modificirend auf das Urehungsvermögen einwirken, so schien es mir zweckmässig, das molekulare Drehungsvermögen für invertirte Zuckerlösungen $n$ äher zu bestimmen und daraus eine neue Tabelle abzuleituen.

7or Ausf ührung dieser Arbeit brauchte ich:

1) Röhr en von ganz genau bestimmter Länge;

2) e in Gefäss von genau bekanntem Inhalt, um die abgewogenen Mengen Zucker darin zu lösen;

3) einen Apparat, um der zu untersuchenden Flüssigkeit, eine gleichmässige und bestimmte Temperatur zu geben.

Ich werde zunüchst die Ausführung dieser Vorarbeiten und Einrichtung der von mir gebrauchten Apparate beschreiben, um hernach zu den eigentlichen Bestimmungen selbst überzugehen.

\section{Bestimmung der Länge der Versuohsröhren.}

Die Länge der Versuchsröhren wurde mittelst eines im Bonner physikalisch-chemischen Laboratorium aufgestellten Kathetometers von Dr. Meyerstein in Göttingen gemessen. Die Theilung des Instrumentes war direct in Millimetern auf einem Silberstreifen aufgetragen; mit Hülfe des Nonius liessen sich 5/100 Mm. direct ablesen und 8/100 Mm. noch gut schätzen, so dass die Länge der verschiedenen Röhren bis auf $\$ / 100 \mathrm{Mm}$. genau bestimmt ist. Für meine Untersuchungen gebrauchte ich 4 Röhren von verschiedener Länge, die theils aus Glas, theils aus Messing angefertigt waren. Die Lüngenbestimmung ergab für dieselben folgende Werthe:

$$
\begin{aligned}
& \text { Röhre } A=199,97 \mathrm{Mm} . \\
& \text { Röhre } B=300,12 \mathrm{Mm} . \mid \text { Messingröhren. } \\
& \text { Röhre } C=227,20 \mathrm{Mm} . \mid \text { Glasröbren. } \\
& \text { Röhre } D=284,65 \mathrm{Mm} . \mid
\end{aligned}
$$


auf das molekulare Drehungsvermögen etc. 239

\section{Bestimmung des Volnmens des Glasgefässes,}

in welchem bestimmte Gewichtsmengen der zu untersuchenden Substanz gelöst wurden, um so Flüssigkeiten von bestimmter Concentration zu erhalten. Ich bediente mich zu diesem Zwecke eines Kölbchens, dessen Hals an einer Stelle so ausgezogen war, dass der Durchmesser der Oeffnung circa $2 \mathrm{Mm}$. betrug. Die engste Stelle des Halses war mit einer Marke versehen. Die Differenz des Gewichtos des Kölbchens, bis zur Marke mit Wasser gefült, und des leeren Kölbchens gab unmittelbar den Inhalt des Gefàsses. Die 'l'emperatur dos Wassers betrug $13,5^{\circ}$, so dass noch der Inhalt $d$ des Kölbchens bei $13,5^{\circ}$ zu

$$
J=112,1118 \text { Cc. ergab. }
$$

Apparat zur Erzengang einer gleichmässigen Temperatur der zu untersuohenden Fltssigkeit.

Da es mir bei meinen Untersuchuugen darauf ankam, die zu untersuchende Flüssigkeit so lange auf der gleichen und gleichmässigen Temperatur zu erhalten, als eine Reihe von Beobachtungen Zeit in Anspruch nahm und mir anderseits von grossem Belang war, die Temperatur der Flüssigkeit wieder möglichst rasch zu wechseln, so construirte ich mir hierzu folgenden Apparat.

Eine gewöhnliche messingene Röhre, wie sie zu saccharimetrischen Proben benutzt werden, wurde mit einem Blechmantel umgeben, so dass dieser überall wasserdicht an die Röhre anschloss und diese möglichst vollständig umschloss. An den beiden entgegengesetzten Enden des Blechmantels waren zwei Oeffinungen angebracht, so dass, wenn man rlurch die eine Oeffinung Wasser zwischen Rohr und Mantel fliessen liess, dasselhe die ganze Röhre zuerst umspielen musste, bevor es an der anderen Oeffnung austreten konnte. Die Oelfinungen $e$ und $d$ (s. Taf. I) dienten zum Einsenken zweier Thermometer bis auf die innere Röhre, so dass nur die Scalen derselben aus dem Apparate hervorragten. Die Thermometer, die ich für den Apparat 
benutzte, waren direct in $1 / 5^{\circ}$ getheilt und liessen $1 / 20^{\circ}$ noch ganz leicht schätzen. In der Mitte bei $a$ hatte die innere Röhre eine Oeffinung; ; in die eine enge Röhre $g$ wasserdicht eingesetzt wurde. Der Röhre $g$ entsprechend besitzt anch der Blechmantel noch eine Oeffinung $f$, durch welche dieselbe nach aussen communicirt; diese Röhre hat den Zweck, dass die beim Erwärmen sich ausdehnende Flüssigkeit der innern Röhre sich hier ausbreiten kann und so in dem Apparat keine Compression stattfindet. Um endlich Wasser von bestimmter Temperatur zwischen Röhre und Mantel zu leiten, wurde neben dem Apparate ein grosses Gefäss mit einem Schlangenrohr aufgestellt. Wurde nun das Gefäss mit Wasser gefüllt und dasselbe durch Erwärmen auf eine bestimmte Temperatur gebracht, so nahm das Wasser, welches man dureh die Schlangenröhre fliessen liess, wenn der Strom nicht all zu stark war, die gleiche Temperatur an. Um also Wasser von bestimmter Temperatur zu haben, brauchte man uur den Apparat mit diesem Schlangenrohr zu verbinden; durch das zwischen Röhre und Mantel fliessende Wasser wurde alsdann die Flüssigkeit in der Röhre selbst erwärmt. Um die möglichste Gleichkeit der Temperatur in der Schlangenröhre und im Gefässe zu erhalten, wurde das Wasser unten in die Schlangenröhre eingeführt, so dass dasselbe möglichst lange in derselben verweilen musste. Durch Einschieben eines Gefässes mit Wasser zwischen die Schlangenröhre und den Wasserzufluss konnte der Fintritt des Wassers in die Schlangenröhre beliebig regulirt werden.

Mit dem so hergestellten Apparate nahm ich zunächst zwei Prüfungen vor, von denen seine Brauchbarkeit abhängig war:

1) lässt sich durch Regulirung des Wasserzuflusses die Temperatur des Wassers zwischen der (die zu untersuchende Flüssigkeit enthaltenden) Röhre und dem Mantel so erhalten, dass die beiden Thermometer, die möglichst weit von einander abstehen und an die Enden der Röhre gerückt sind, die gleiche Temperatur zeigen; 
auf das molekulare Drehungsvermögen etc. 241

2) nimmt die Flïssigkeit im Innern der Röhre rasch genug die Temperatur des sie umspülenden Wassers an.

Zur Beurtheilung der ersten Frage wurde längere Zeit Wasser von bestimmter und gleicher Temperatur (was sich leicht dadurch erreichen liess, dass man die Temperatur des Wassers in dem Gefäss immer auf der gleichen Höhe erhielt und den Wasserzufluss immer gleich stark hatte) durch den Apparat geleitet. Es ergab sich hierbei, dass das von der Einflussstelle des Wassers entferntere Thermometer nie ganz genau die gleiche Temperatur zeigte, wie das Thermometer, wo das Wasser in den Apparat eintrat, indem sich der Apparat von aussen her immer etwas abkühlte. Doch kann bei hinlänglich starkem Wasserzufluss die Temperatur so regulirt werden, dass die Differenz der beiden Thermometerangaben höchstens $1 / \mathrm{s}^{0}$ beträgt; um diesen Fehler zu eliminiren, wurde bei den spätern Beobachtungen die Temperatur an beiden Thermometern abgelesen und aus den Ablesungen das arithmetische Mittel genommen.

Um über den zweiten Punkt im Klaren zu sein, wurde die mittlere Röhre mit Wasser gefüllt und bis in die Mitte derselben ein Thermometer, von gleichem Gange wie die beiden übrigen, eingeschoben. Wurde nun durch Einleiten von warmem Wasser zwischen Röhre und Blechmantel die Röhre erwärmt, so zeigte sich, dass ein Paar Minuten nachdem die Thermometer $A$ und $B$ im dem Mantel eine constante Temperatur angezeigt hatten, auch das Thermometer in der Röhre einen bestimmten Stand eingenommen hatte, und zwar zeigte dieses Thermometer eine Temperatur, die gerale das arithmetische Mittel der Angaben der Thermometer $A$ und $B$ war. Es war somit der Apparat in das Saccharimeter so eingeschoben, dass die mittlere Röhre genau mit der Richtung des polarisirten Lichtstrables zusammenfiel, vollkommen ausreichend für die Zwecke, die derselbe erfüllen sollte.

Meine folgenden Bestimmungen der Abhängigkeit der Drehung der Polarisationsebene von der Temperatur Jouru. f. prakt. Chemie [2] Bd. 2. 
beziehen sich auf Zuckerlösungen (sowohl reine als invertirte), auf Lösungen von Kampher in Alkohol und auf Weinsäurelösungen. Die Beobachtungen bei Zuckerlösungen geschahen sowohl mit dem Soleil-Duboscq'schen Instrument, als mit dem Polaristrobometer von Wild. Für die Kampher- und Weinsäurelösungen konnte natürlich nur das Wild'sche Instrument gebraucht werden, da sich das Soleil'sche Instrument bei der Anwendung des Quarzcompensators nur zur Beobachtung solcher Flüssigkeiten gebrauchen lässt, die das gleiche Dispersionsvermögen wie der Quarz besitzen.

Das erse Instrument von Duboseq in Paris war mit Doppelplatte und Quarzcompensator versoben, an der linearen Scale war der Nullpunkt durch eine optisch inactive Substanz, z. B. Wasser, der Punkt 100 dagegen durch die Ablenkung, welche eine Platte rechts drehenden Bergkrystalls von genau $1 \mathrm{Mm}$. Dicke hervorbringt, bestimmt, so dass bei dem bekanntlich gleichen Dispersionsvermögen des Quarzes mit Zucker die abgelesenen Theilstriche direct die Procente an Zucker angaben, wenn man die abgewogene Menge Zucker in $100 \mathrm{Cc}$. Wasser löste und die erhaltene Lösung in einer Röhre von $200 \mathrm{Mm}$. Lünge untersuchte. Zur Ablesung der Scala, die an der einen Quarzplatte des Quarzcompensators befestigt war, befand sich an der zweiten Quarzplatte ein einfacher Index, der beim Bewegen der letztern längs der Scala glitt. Die Soala war direct so eingetheilt, dass jeder Theilstrich genau 1 p.C. Zucker in der erwähnten Lösung angab; mit Hülfe des Index liessen sich aber noch leicht $1 / 10$ Theilstriche schätzen. Die Beobachtungen geschahen sämmtlich mit einer gewöhnlichen Gaslampe.

Das Wil d'sche Polaristroborneter ${ }^{1}$ ), welches ich zu meinen Untersuchungen gebrauchte, war ein solches grösserer Form vom Optiker Hoffmann in Paris. Es hatte das Instrument einen Theilkreis von 0,1 Meter Durchmesser; die Theilung war direct in $1 / 2$ Grade, und mit Hülfe des

3) Wild, Polaristrobometer. 
auf das molekulare Drehungsvermögen etc. 243

Nonius las man direct 0,02 Grade ab. Bei den Beobachtungen diente als Lichtquelle stets eine Bunsen'sche Gasflamme, in der kohlensaures Natron mit Hülfe eines Platindrahtes verflüchtigt wurde, so dass sich alle meine Beobachtungen mit diesem Instrumente auf den gelben, der Fraunhofer'schen Linie $D$ gensu entaprechenden Lichtstrahl beziehen. Die Einstellung auf das Versehwinden der Interferenzstreifen gesshah an zwei gegenüber liegenden Quadranten.

\section{Zucker.}

A. Reine Zuckerlösungen.

Da es mir nicht darauf ankam, das molekulare Drehungsvermögen einer Zuckerlösung für eine bestimmte Temperatur zu ermittelu, Werthe, die bereits von verschiedenen Forschern sowohl mit dem Soleil'schen als dem Wild'schen Apparat ganz genau bestimmt wurden, sondern rielmehr um das Variiren dieses Drehungsvermögens mit der Temperatur, so stellte ich mir eine beliebige Lösung reinen Zuckers dar und untersuchte sie in einer der früher beschriebenen Röhren bei verschiedenen Temperaturen; doch stellte sich bald heraus, dass bei geringen Temperaturschwankungen die Aenderungen im Drehungsvermögen so minim ansfielen, dass sowohl die Beobachtungsfehler mit dem Soleil'schen, als dem Wild'schen Apparat grösser waren. Es war mir daher wahrscheinlich, dass, wenn überhaupt Sehwankungen im Drehungsvermögen vor sich gehen, diese nur von der Ausdehnung der Flüssigkeit beim Erwärmen herrührten.

Das molekulare Drehungsvermögen [ $\alpha]$ einer activen Substanz ist durch irgend eine Farbe nach Biot durch die Gleichung definirt:

$$
[\alpha]=\frac{a}{l \cdot \varepsilon \cdot \delta,}
$$

wo $l$ die Länge der eingeschalteten Substanz (sei es allein oder vermengt mit einer inactiven Substanz), die eine Drehung von \% Graden für die betreffende Farbe hervor- 
244 Tuchschmid: Einfluss der Temperatur

bringt, $\delta$ das specifische Gewicht dieser Substanz, resp. des Gemisches und $\varepsilon$ das Verhältniss des Gewichtes der activen Substanz zum Gewicht des ganzen Gemisches. Es ist somit

$$
\alpha=[\alpha] l . \varepsilon . \delta .
$$

Nehmen wir $[\alpha]$ für verschiedene Temperaturen constant an, so kann sich in der Formel für $\alpha$ nur der Werth von $\delta$ mit der Temperatur ändern, wenn wir die Aenderungen von $l$ ausser Acht lassen, die bei den geringen Temperaturdifferenzen, um welche es sich hier handelt, äusserst klein sein würden. Berechnen wir nun nach dieser Formel die Werthe für $\alpha_{t}$ bei zwei Temperaturen, die weit aus einander liegen, für eine "Zuckerlösung, bei der $\varepsilon=0,249$, so brauchen wir nur an die Stelle von $\delta$ die diesen Temperaturen für $\varepsilon$ entsprechenden Werthe von $\delta$ einzusetzen. Die Werthe von $\delta$ sind nach Gerlach ${ }^{2}$ )

$$
\begin{aligned}
& \text { bei } 10^{\circ}=1,10206 \text {, } \\
& \text {, } 27,5^{\circ}=1,09656 \text {. }
\end{aligned}
$$

Nimmt man ferner für $[\alpha]$ den Werth $66,417^{\circ}$ und für $l$ den Werth $100 \mathrm{Mm}$., so ergeben sich für $\alpha_{1}$ bei $10^{\circ}$ und $\alpha_{2}$ bei $27,5^{\circ}$ folgende Werthe:

$$
\begin{aligned}
\alpha_{1} & =18,226^{\circ}, \\
\alpha_{2} & =18,135^{\circ} . \\
\text { Differenz } & =0,091^{\circ} \text { der Kreistheilung. }
\end{aligned}
$$

Bei den Beobachtungen, die ich mit obiger Lösung anstellte, ergab sich:

$$
\begin{aligned}
\alpha \text { bei } 10,4^{\circ} & =18,243 \pm 0,3 \text { (Mittel aus } 40 \text { Beobacht.) } \\
\alpha \text { bei } 40,5^{\circ} & =18,077 \pm 0,3(”, ", ", \\
\text { Differenz } & =0,166^{\circ} \text { Kreistheilung. }
\end{aligned}
$$

Diese Zahlen sind das Mittel aus den Beobachtungen mit dem Soleil'schen und dem Wild'schen Apparat.

Nach den Beobachtungen beträgt somit die Differenz in der Drehung für die genannte Zuckerlösung bei der

1) Bolley, Handb. d. chem.techn. Untersuchungen, S. 442. 
auf das molekulare Drehungsvermögen etc. 245

Aenderung der Temperatur um $30,1^{\circ}=0,166^{\circ}$. Für die gleiche Temperaturdifferenz würde der Unterschied in der Drehung nach der Formel berechnet $0,157^{\circ}$ betragen. Die durch den Versuch ermittelten Zahlen stimmen so gut mit den berechneten Zahlen, dass es keinem Zweifel unterliegen kann, dass die Aenderung im Drehungsvermögen einer reinen Zuekerlösung mit der Temperatur nur von der gleichzeitig eintretenden Aenderung im specifischen Gewicht der Flüssigkeit herrührt. Es lässt sich demnach mit Bestimmtheit der Satz aussprechen:

Das molekulare Drehungsvermögen reiner Zuckerlösungen ist von der Temperatur unabhängig.

B. Invertirte Zuckerlösungen.

Die directe Polarisation von "'uckerlösungen giebt den Gehalt derselben nur dann richtig an, wenn in der vorhandenen Lösung keine Substanzen vorhanden sind, die ebenfalls drehend auf die Polarisationsebene einwirken. Um den Fehler, der durch solche Substanzen hervorgerufen wird, zu eliminiren, wird die Inversion der Zuckerlösungen vorgenommen, wodurch der Rohrzucker in linksdrehtenden Invertzucker übergeführt wird. Diese Methode stïtzt sich darauf, dass von den verschiedenen optisch activ wirkenden Substanzen, die in dem Zucker enthalten sein können, nur der rechtsdrehende Rohrzucker bei der Inversion eine Umwandlung erleidet. Nach den Versuchen von Clerget zeigte eine Lösung von $16,35 \mathrm{Grm}$. reinen Zuckers zu 100 Co., die am Soleil'schen Saccharimeter eine Rechtsdrehung um $100^{\circ}$ hervorbringt, nach der Inversion, wenn die Beobachtung bei $0^{\circ}$ gemacht wurde, eine Drehung von $44^{\circ}$ nach links, so dass also durch die Inversion eine Drehungsverminderung um $144^{\circ}$ stattgefinden hat. Für eine Temperaturerhöhung von je $1^{\circ}$ wird die Linksablenkung der Lösung um 0,5 Theilstriche kleiner. Bedeutet $S$ die Summe der Saccharimeterablesungen vor und nach der Inversion, $T$ die Temperatur, bei der die invertirte Zuckerlösung untersucht 


\section{Tuchschmid: Einfluss der Temperatur}

warde und $R$ den Procentgehalt der Lösung, so lässt sich die Proportion aufstellen:

$$
144-1 / 2 T: 100=S: R,
$$

oder

$$
\begin{aligned}
288-T: 200 & =\delta: R \\
R & =\frac{200 S}{288-T} .
\end{aligned}
$$

Dies ist die bekannte Formel, auf die Clerget seine Tabelle gründet.

Es handelte sich nun bei meinen Bestimmungen darum:

1) Dreht die oben erwähnte Zuckerlösung nach der Inversion die Polarisationsebene genau um $44^{\circ}$ nach links?

2) Ist die Drehung bei verschiedenen Temperaturen der Zunahme der Temperatur genau proportional, wie es Clerget bei Aufstellung seiner Formel annimmt?

Diese Untersuchungen fanden sowohl mit dem Soleil'schen als dem Wild'schen Instrument statt.

A. Beobachtungen mit dem Soleil'schen Apparat. Um zunächst den Einfluss der Temperatur auf das Drehungsvermögen invertirter Zuckerlösungen zu ermitteln, wurde eine beliebige Quantität reinen Zuckers, in Wasser gelöst, mit concentrirter Salzsäure in der bekannten Weise durch Erhitzen auf $68^{\circ}$ während circa 10 Minuten invertirt und diese Lösung nach dem Erkalten in dem früher beschriebenen und für diese saure Flüssigkeit in Glas ausgeführten Apparat untersucht. Bei jeder Temperatur wurden mindestens 20 Beobachtungen gemacht, die um $\pm 0,2- \pm 0,5$ Theilstriche der Soleil'schen Scala differirten und alsdann aus denselben das arithmetische Mittel genommen. Die Fehler, die hierdurch in der Ablesung entstehen, können nach den Untersuchungen von Prof. Landolt höchstens 0,02 Theilstriche betragen. Bei vielen Temperaturen wurden die Beobachtungen öfters wiederholt und aus den ermittelten Ablenkungen das Mittel genommen. Um allfällige Schwankungen der Temperatur der Lösung 
auf das molekulare Drehungsvermögen etc. 247

während des Versuches möglichst zu eliminiren, wnrden an beiden Thermometern vor und nach dem Versuch die Temperaturen abgelesen und aus den Angaben das Generalmittel genommen. Es ergaben sich hierbei folgende Werthe:

\begin{tabular}{|c|c|c|c|c|c|c|c|c|}
\hline & & Temper & aturen & & & \multirow{3}{*}{ 这 } & \multirow{3}{*}{$\begin{array}{c}\text { Mittel } \\
\text { aus } \\
\text { sämmtlichen } \\
\text { Einstellungen. }\end{array}$} & \multirow{3}{*}{$\begin{array}{c}\text { Zahl der } \\
\text { Ein- } \\
\text { stellungen. }\end{array}$} \\
\hline \multicolumn{2}{|c|}{ vor dem } & rench. & \multirow{2}{*}{$\frac{\text { nach }}{\text { A }}$} & \multicolumn{2}{|c|}{ dem Versueh. } & & & \\
\hline $\mathbf{A}$ & B & Mittel. & & B & Mittel. & & & \\
\hline 11,9 & 11,7 & 11,8 & 12,1 & 11,9 & 12,0 & 11,9 & $33,20 \pm 0,5$ & 20 \\
\hline 23,2 & 23,2 & 23,2 & 29,2 & 23,2 & 23,2 & 23,2 & $28,45 \pm 0,5$ & 21 \\
\hline 12,2 & 12,2 & 12,2 & 12,4 & 12,4 & 12,4 & 12,3 & $33,01 \pm 0,3$ & 21 \\
\hline 36,8 & 36,8 & 36,8 & 36,6 & 36,6 & 36,6 & 36,7 & $21,55 \pm 0,5$ & 23 \\
\hline 34,8 & 34,8 & 34,8 & 34,5 & 34,5 & 34,5 & 34,65 & $22,66 \pm 0,3$ & 22 \\
\hline 12,0 & 12,2 & 12,1 & 12,4 & 12,2 & 12,3 & 12,2 & $33,09 \pm 0,3$ & 19 \\
\hline 18,6 & 18,6 & 18,6 & 18,8 & 18,8 & 18,8 & 18,7 & $30,54 \pm 0,3$ & 21 \\
\hline 17,8 & 17,9 & 17,85 & 18,2 & 18,0 & 18,1 & 18,0 & $30,85 \pm 0,3$ & 23 \\
\hline 19,9 & 19,8 & 19,8 & 20,5 & 20,3 & 20,4 & 20,1 & $29,96 \pm 0,3$ & 22 \\
\hline 34,6 & 34,4 & 34,5 & 34,2 & 34,0 & 34,1 & 34,3 & $22,81 \pm 0,3$ & 19 \\
\hline 28,0 & 28,0 & 28,0 & 27,6 & 27,6 & 27,6 & 27,75 & $26,18 \pm 0,4$ & 19 \\
\hline 14,5 & 14,7 & 14,6 & 14,6 & 14,4 & 14,5 & 14,45 & $32,12 \pm 0,4$ & 21 \\
\hline 26,7 & 26,7 & 26,7 & 26,9 & 26,9 & 26,9 & 26,8 & $26,65 \pm 0,5$ & 23 \\
\hline 16,8 & 16,8 & 16,8 & 17,4 & 17,2 & 17,3 & 17,05 & $31,29 \pm 0,3$ & 19 \\
\hline 21,6 & 21,6 & 21,6 & 21,6 & 21,6 & 21,6 & 21,6 & $29,26 \pm 0,4$ & 22 \\
\hline 30,8 & 30,6 & 30,7 & 30,0 & 30,0 & 30,0 & 30,3 & $24,82 \pm 0,3$ & 21 \\
\hline 32,8 & 32,8 & 32,8 & 32,8 & 32,8 & 32,8 & 32,8 & $23,65 \pm 0,4$ & 19 \\
\hline 33,4 & 33,4 & 33,4 & 33,2 & 33,2 & 33,2 & 33,3 & $23.30 \pm 0,5$ & 20 \\
\hline 35,4 & 35,6 & 35,5 & 35,6 & 36,0 & 35,8 & 35,6 & $22,25 \pm 0,3$ & 19 \\
\hline 41,8 & 41,8 & 41,8 & 41,8 & 41,8 & 41,8 & 41,8 & $19,05 \pm 0,2$ & 10 \\
\hline 10,0 & 10,0 & 10,0 & 10,0 & 10,0 & 10,0 & 10,0 & $33,97 \pm 0,3$ & 15 \\
\hline 25,4 & 25,4 & 25,4 & 25,2 & 25,2 & 25,2 & 25,3 & $27,34 \pm 0,4$ & 18 \\
\hline 20,2 & 20,0 & 20,1 & 19,8 & 19,6 & 19,7 & 19,9 & $29,99 \pm 0,2$ & 20 \\
\hline 34,0 & 34,2 & 34,1 & 33,8 & 34,0 & 33,9 & 34,0 & $22,89 \pm 0,4$ & 19 \\
\hline 37,0 & 37,1 & 37,05 & 37,5 & 37,5 & 37,5 & 37,3 & $21,33 \pm 0,3$ & 19 \\
\hline 39,4 & 39,4 & 39,4 & 39,6 & 39,6 & 39,6 & 39,5 & $20,24 \pm 0,4$ & 20 \\
\hline 37,5 & 37,5 & 37,5 & 37,5 & 37,5 & 37,5 & 37,5 & $21,27 \pm 0,4$ & 20 \\
\hline 32,0 & 31,8 & 31,9 & 32,4 & 32,4 & 32,4 & 32,2 & $23,92 \pm 0,3$ & 20 \\
\hline 7,0 & 7,6 & 7,3 & 7,0 & 7,6 & 7,3 & 7,3 & $35,12 \pm 0,5$ & 18 \\
\hline 4,0 & 4,0 & 4,0 & 4,0 & 4,0 & 4,0 & 4,0 & $36,35 \pm 0,4$ & 19 \\
\hline
\end{tabular}

B. Beobachtungen mit dem Wild'schen Instrument. Die gleiche Lösung wurde in einer ähnlichen Röhre aus Glas am Wild'schen Instrument untersucht und dabei gefunden: 
Tuchschmid: Einfluss der Temperatur

\begin{tabular}{|c|c|c|c|c|c|c|c|c|}
\hline vor & & $\begin{array}{l}\text { Tempe } \\
\text { such. }\end{array}$ & $\begin{array}{l}\text { raturen } \\
\text { nach }\end{array}$ & $\operatorname{dem} \mathrm{v}$ & rsuch. & 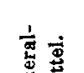 & $\begin{array}{l}\text { Mittel } \\
\text { aus }\end{array}$ & $\begin{array}{l}\text { Zahl der } \\
\text { Ein- }\end{array}$ \\
\hline $\mathbf{A}$ & B & Mittel. & $\Lambda$ & B & Miftel. & & Einstellungen. & \\
\hline 32,2 & 32,2 & 32,2 & 32,2 & 32,2 & 32,2 & 32,2 & $-7,62 \pm 0,03$ & 10 \\
\hline 34,2 & 33,8 & 34,0 & 34,0 & 33,6 & 33,8 & 33,9 & $\ldots \quad 7,33 \pm 0,04$ & 10 \\
\hline 7,5 & 8,2 & 7,9 & 8,5 & 8,2 & 7,9 & 7,9 & $-11,31 \pm 0,02$ & 10 \\
\hline 19,9 & 19,9 & 19,9 & 20,2 & 20,2 & 20,2 & 20,05 & $-9,65 \pm 0,01$ & 10 \\
\hline 21,2 & 21,2 & 21,2 & 21,2 & 21,2 & 21,2 & 21,2 & $-9,48 \pm 0,04$ & $\theta$ \\
\hline 27,8 & 27,8 & 27,8 & 27,8 & 27,8 & 27,8 & 27,8 & $-8,40 \pm 0,04$ & 11 \\
\hline 30,8 & 30,8 & 30,8 & 30,8 & 30,8 & 30,8 & 30,8 & $-7,79 \pm 0,03$ & 10 \\
\hline 19,0 & 19,0 & 19,0 & 19,0 & 19,0 & 19,0 & 19,0 & $-9,80 \pm 0,02$ & 12 \\
\hline 24,8 & 24.8 & 24,8 & 24,4 & 24,4 & 24,4 & 24,6 & $-8,95 \pm 0,04$ & 19 \\
\hline 29,8 & 23,6 & 23,7 & 23,8 & 23,6 & 23,7 & 23,7 & $-9,06 \pm 0,03$ & 14 \\
\hline 26,0 & 26,0 & 26,0 & 26,0 & 26,0 & 26,0 & 26,0 & $-8,69 \pm 0,04$ & 11 \\
\hline 14,1 & 14,2 & 14,2 & 14,2 & 14,2 & 14,2 & 14,2 & $-10,40 \pm 0,01$ & 13 \\
\hline 39,8 & 39,8 & 39,8 & 39,8 & 39,8 & 39,8 & 39,8 & $-6,41 \pm 0,04$ & 10 \\
\hline 36,0 & 36,0 & 36,0 & 36,0 & 36,0 & 36,0 & 36,0 & $-7,00 \pm 0,03$ & 9 \\
\hline 16,0 & 16,0 & 16,0 & 16,0 & 16,0 & 16,0 & 16,0 & $-10,18 \pm 0,04$ & 10 \\
\hline 10,6 & 10,6 & 10,6 & 10,6 & 10,6 & 10,6 & 10,6 & $-10,91 \pm 0,03$ & 11 \\
\hline 6,0 & 6,0 & 6,0 & 6,0 & 6,0 & 6,0 & 6,0 & $-11,60 \pm 0,04$ & 14 \\
\hline
\end{tabular}

Tragen wir die so erbaltenen Werthe für die $\mathrm{Ab}$ lenkungen des polarisirten Tichtstrahles einer invertirten Zuckerlösung fiur verschiedene Temperaturen in ein Coordinatennetz so ein, dass wir anf der Abscissenaxe die Temperaturen, auf der Ordinatenaxe die zugehörigen Ablenkungswinkel sowohl beim Soleil'schen '), als beim Wild'schen ${ }^{3}$ ) Apparat haben, so ergeben sich fiir die beiden Instrumente zwei beinahe vollkommen zusammenfallende Curven, zum vollkommenen Beweis der Richtigkeit der gemachten Beobachtungen. Diese Curven weichen von der gerarien Ijinie so wenig $a b$, dass diese Abweichung ihren Grund in der Aenderung des specifischen Gewichtes der Lösung bei den verschiedenen Termperaturen haben knnnte. Tch ermittelte zu diesem Zwecke die specilischén Gewichte dieser Lösung bei verschiedenen Temperaturen and erhielt dafür folgende Werthe:

1) Sieho Taf. II.

) Siebe Taf. III. 
auf das molekulare Drehungsvermögen etc. 249

$$
\begin{array}{rlrl}
6,4^{0} & =1,08068 & 24,6^{0} & =1,07482 \\
12,2^{0} & =1,07867 & 26,2^{\circ} & =1,07325 \\
15,0^{0} & =1,07788 & 29,6^{0} & =1,07233 \\
17,4^{0} & =1,07725 & 35,4^{0} & =1,07075 \\
19,4^{0} & =1,07670 & 42,6^{0} & =1,06788 .
\end{array}
$$

Diese Werthe in entsprechender Weise in ein Coordinatennetz eingetragen, geben in der That eine Curve, die in entsprechender Weise verläuft, wie die beiden vorher genannten Curven. Es konnte daher keinem Zweifel unterliegen, dass die Aenderung des Drehungsvermögens einer invertirten Zuckerlösung proportional mit der Zunahme der Temperatur vor sich gehe. Will man daher für die untersuchte Lösung eine Interpolationsformel berechnen, aus der man für irgend eine Temperatur den Drehungswinkel $\alpha$ berechnen kann, so kann man dieser die Form geben

$$
\alpha=x+y t
$$

worin $t$ die Temperatur und $x$ und $y$ zwei constante Grössen repräsentiren.

Diese Werthe für $x$ und $y$ nach der Methode der kleinsten Quadrate bestimmt, ergeben:

$$
\begin{gathered}
\text { beim Soleil'schen Apparat } \\
x=38,982 \quad y=-0,4694, \\
\\
\text { beim Wild'schen Apparat } \\
x=19,4781 \quad y=-0,2306 .
\end{gathered}
$$

Um ferner einen Punkt der Curve genau zu bestimmen, wurden genau $16,1356 \mathrm{Grm}$. reinen und längere Zeit unter der Luftpumpe getrockneten Candiszuckers in Wasser gelöst und in das mit Marke versehene Kölbchen von $112,1118 \mathrm{Ce}$. Inhait nach der Invertirung genau gefüllt. Diese lı̈̈sung würde in einer Röhre von $227,2 \mathrm{Mm}$. Länge vor der Inversion eine Rechtsdrehung um $100^{\circ}$ der Soleil'sohen Scala erzeugt haben, und gab so berechnet auf eine Röhre von $200 \mathrm{Mm}$. Länğe bei $21^{\circ}$ im Mittel ans 40 Beobachtungen eine Drehung wach links von $33,539^{\circ} \pm 0,4$. 


\section{Tuchschmid: Einfluss der Temperatur}

Nach dem Vorigen hängt der Drehungswinkel [a] einer invertirten Zuekerlösung von der Temperatur in der Weise $a b$, dass

$$
\alpha=38,982+(-0,4694 t) \pm 0,3 .
$$

$\mathrm{Da}$ also eine Lösung von 16,35 Grm. reinen Zuckers in $100 \mathrm{Cc}$. nach der Inversion eine Ablenkung von 33,539 a bewirkt, wenn die Beobachtung bei $21^{\circ}$ geschah, so lässt sich darnach leicht für diese Lösung die Abhängigkeit des Drehungswinkels $\alpha_{t}$ von der Temperatur berechnen:

$$
\alpha_{t}=44,16035+(-0,50578 t) \text {. }
$$

Wird $t=0$, so ist $\alpha_{t}=44,16035$, d. h. eine Lösung von 16,35 Grm. reinen Zuckers zu $100 \mathrm{Cc}$. gelöst, giebt nach der Inversion, wenn die Beobachtung bei $0^{\circ}$ gemacht wurde, eine Drehung von $44,16035^{\circ}$ nach links; diese Drehung nimmt bei dieser Flüssigkeit für eine Temperaturerhöhung von je $1^{\circ}$ um 0,50578 Theilstriche der Soleil'schen Scala ab. Es ergaben sich so für $x$ und $y$ Werthe, die ziemlich genau mit den früher von Clerget ermittelten übereinstimmten.

Für irgend eine Lösung lässt sich die Abhängigkeit des Drehungswinkels $\alpha_{t}$ von der Temperatur durch die Gleichung definiren

$$
\alpha_{t}=\alpha_{0}-\frac{\alpha_{0} .0,50578 t}{44,16035},
$$

wenn $\alpha_{0}$ der Ablenkungswinkel bei $0^{\circ}$ wäre.

$$
\alpha_{0}=\frac{\alpha t .44,16035}{44,16035-0,50578 t} .
$$

Die gleiche Betrachtung, auf das Wild'sche Instrument angewandt, würde zu der Formel führen:

$$
\alpha_{0}=\frac{a_{\mathrm{t}} .9,5911}{9,5911-0,10985 \bar{t}} .
$$

Fine Lösung von $16,35 \mathrm{Grm}$. reinen Zuckers in $100 \mathrm{Cc}$., die bei der directen Polarisation eine Ablenkung von $100^{\circ}$ zeigt, giebt nach der Inversion bei $0^{0}$ eine Linksablenkung um 44,16035 ${ }^{\circ}$ der Soleil'schen Seala; bei der Temperaturerhöhung um je $1^{0}$ nimmat diese um $0,50578^{\circ} \mathrm{ab}$; es ist somit die Drehungsverminderung, die durch die Inversion 
auf das molekulare Drehungsvermögen etc. 251 erfolgte, bei $t^{0}=144,16035-0,50578 t$. Bedeutet $S$ die Summe der Saccharimeterablesungen vor und nach der Inversion, $\boldsymbol{T}$ die Temperatur, bei der die invertirte Zuckerlösung beobachtet wurde, und $R$ den gesuchten Procentgehalt einer Rohrzuckerlösung, so gilt die Proportion:

$$
\begin{gathered}
144,16035-0,50578 T: 100=S: R \\
R=\frac{100 S}{144,16085-0,50578 T} .
\end{gathered}
$$

Für das Wild'sche Instrument:

$$
R=\frac{S 21,7189}{31,310-0,10985 T} .
$$

Mit Hülfe dieser Formeln lässt sich die Clerget'sche Tabelle für das Soleil'sche ${ }^{1}$ ) und Wild'sche Instrument umrechnen.

Um die Menge des in einem Zucker enthaltenen Invertzuckers zu erkennen, kann man in folgender Weise verfahren:

Da 16,35 Grm. Rohrzucker zu $100 \mathrm{Cc}$. beim Invertiren eine Flüssigkeit geben, die bei $t^{0}$ eine Linksdrehung von 44,16035-0,50578 $t$ hervorbringt, so entspricht diese Drehung, indem 171 Theile Rohrzucker durch die Behandlung mit Säure 180 Theile Invertzucker liefern, einem Gehalte von 17,21 Grm. Invertzucker in $100 \mathrm{Cc}$. Ist $A$ das Ergebniss der directen Polarisation, $R$ der durch Inversion gefundene Rohrzuckergehalt und $J$ die gesuchte Menge des Invertzuckers in Grammen, so hat man:

$$
\begin{gathered}
44,16035-0,50578 T: 17,21=R-A . J, \\
J=\frac{17,21(R-A)}{44,16085-0,50578 \mathrm{~T}} .
\end{gathered}
$$

In ähnlicher Weise ist $J$ für das Wild'sche Instrument

$$
J=\frac{17,21(\boldsymbol{R}-\boldsymbol{A})}{9,5911-0,10985 \mathrm{~T}} .
$$

1) Siehe Anhang: Tafel zur Analyse zuekerhaltiger Substanzen. 


\section{Kampher.}

Zur Untersuchung der Drehung des Kamphers wurde derselbe in Alkohol gelöst und diese Lösung in entsprechender Weise, wie die Zuckerlösungen, am Wild'schen Apparat untersucht. Eine solche alkoholische Kampherlösung zeigte, bei $10^{\circ}$ und bei $40^{\circ}$ boobachtet, eine kaum merkliche Drehungsverschiedenheit, so dass das Drehungsvermögen von Kampher als von der Temperatur unabhängig betrachtet werden kann. Fruiher schon hatte Arndtsen () nachgewiesen, dass die Rotationskraft der Kampherlösungen mit der Concentration varïre und zwar so, dass diese durch eine lineare Gleichung ausgedrückt werden kann. Prof. Landolt, der später die gleichen Bestimmungen wiederholte und die Rotationskraft aus den Brechungsexponenten der Lösungen berechnete, kam hierbei auf so verschiedene Zahlen, dass ich diese Bestimmungen mit Hülfe des Wild'schen Instrumentes ausführte, natürlich nur für die Strahlen der Brechbarkeit $D$.

Verschiedene alkoholische Kampherlösungen, die ich mir $z u$ dem Ende herstellte, gaben auf eine Röhre von $100 \mathrm{Mm}$. Länge reducirt folgende Ablenkungen:

( $q$ sei der Gehalt an Kampher in 1 Theil der Lösung)

$$
\begin{array}{rlrl}
\multicolumn{1}{c}{\quad \text { Rechtsdrehung. }} & & q & \text { Reohtsdrehung. } \\
0,0644 & =2,8703 & 0,05 & =2,183 \\
0,02676 & =1,075 & 0,080=3,500 \\
0,0133 & =0,414 & 0,040=1,717 \\
0,0064=0,119 . & &
\end{array}
$$

Diese Werthe in ein Coordinatennetz ${ }^{3}$ ) eingetragen, liefern Punkte, die alle in einer Geraden liegen; man kann daher die Abhängigkeit des Drehungswinkels $\varrho$ von der Concentration fïr den Strahl $D$ durch die Gleichung ausdriicken:

$$
\varrho=A+B \cdot q
$$

5) Compt. rend. 47, 738 oder Pogg. Ann. 105, 312.

2) Siehe Taf. IV. 
auf das molekulare Drehungsvermögen etc. 253

Berechnet man nach der Methode der kleinsten Quzdrate die Werthe für $A$ und $B$, so ergiebt sich:

$$
\begin{aligned}
& A=-0,1765, \quad B=46,62 . \\
& \rho=-0,1765+46,62 . q \cdot \pm 0,04 .
\end{aligned}
$$

Für $q_{1}=0,0799$ und $q_{2}=0,1598$ sind $\rho_{1}$ und $\rho_{2}$

$$
\rho_{1}=3,548 \quad \rho_{2}=7,274
$$

\section{Weinstinre.}

Die Weinsäure dreht den polarisirten Lichtstrahl nach rechts und es hängt ihr molekuläres Drehungsvermögen nicht nur von der Concentration der zu untersuchenden Lösungen sondern auch von der Temperatur ab.

Es handelt sich zunächst um Ermittlung des Gesetzes, nach welchem die Aenderung des Drehungsvermögens mit der Temperatur vor sich geht. Zu diesem Zwecke wurden 32,70 Grm. reine und vollkommen unter der Luftpumpe getrocknete Weinsäure in so viel Wasser gelöst, dass in $100 \mathrm{Cc}$. der Lösung 29,1673 Grm. Weinsäure enthalten waren. Diese Lösung gab, in einer Röhre von 284,65 Mm. Länge untersucht, bei verschiedenen Temperaturen folgende Ablenkungen :

$\begin{array}{cccc}\text { Temperatur. } & \text { Ablenkung. } & \text { Temperatur. } & \text { Ablenkung. } \\ 8^{\circ} & 8,05 & 30^{\circ} & 9,78 \\ 13^{\circ} & 8,16 & 32^{\circ} & 9,87 \\ 18,2^{\circ} & 8,46 & 33^{\circ} & 9,96 \\ 22,5^{\circ} & 8,87 & 36^{\circ} & 10,07 \\ 25^{\circ} & 9,26 & 39^{\circ} & 10,26 \\ 27,8^{\circ} & 9,75 & 42^{\circ} & 10,46 .\end{array}$

Diese Werthe in ein Coordinatennetz ${ }^{1}$ ) eingetragen geben keine fortlaufende Curve, so dass sich die Abhängigkeit der Drehung der Weinsäurelösungen von der Temperatur nur durch eine höchst complicirte Formel ausdrücken liesse. Aus dem Verlaufe der Curve ersieht man aber:

1) Siehe Taf. IV. 
254 Tuchschmid: Einfluss der Temperatur etc.

1) Die Drehung der Weinsäurelösungen nimmt zu mit dem Wachsen der 'T'emperatur.

2) Es nimmt das Drehungsvermögen von niedern Temperaturen bis zu 27,8 ${ }^{\circ}$ rasch zu; von diesem Punkt an geht die Zunahme der Drehung langsamer vor sich.

Um endlich die Abhängigkeit des molekularen Drehungsvermögens von der Concentration zu ermitteln, wurden verschiedene Weinsäurelösungen bei der gleichen Temperatur von 15,8 untersucht; dieselben gaben nach der Reduction auf eine Röhre von $100 \mathrm{Mm}$. Länge folgende Ablenkungen:

$\begin{array}{cccc}q & \text { Ablenkung. } & q & \text { Ablenkung. } \\ 0,292 & 2,96 & 0,270 & 2,71 \\ 0,146 & 1,64 & 0,190 & 2,02 \\ 0,219 & 2,28 & 0,111 & 1,33 \\ 0,250 & \mathbf{2 , 5 5} . & & \end{array}$

Diese Werthe in ein Coordinatennetz ${ }^{1}$ ) eingetragen, geben Punkte, die in einer Geraden liegen. Man kann daher der Gleichung, die die Abhängigkeit einer Weinsäurelösung von der Concentration angiebt, die Form geben:

$$
\rho=A+B \cdot q \text {. }
$$

Berechnet man die Werthe der Constanten $A$ und $B$, so ergiebt sich:

$$
\begin{aligned}
& A=0,382 \quad B=8,87 \\
& o=0,381+8,67 \cdot q \cdot \pm 0,02 .
\end{aligned}
$$

Diese Gleichung gilt natürlich nur für die Strahlen von der Brechbarkeit der Fraunhofer'schen Linie $D$.

Die vorstehende Arbeit wurde im chemisch-physikalischen Laboratorium des Hrn. Prof. Landolt ausgeführt. Es sei mir hier gestattet, meinem hochverehrten Lehrer für den mir in reichem Maasse zu Theil gewordenen Ratb meinen bleibenden Dank auszusprechen.

1) Siehe Taf. VI. 